\title{
Desafíos de la Responsabilidad Social Corporativa en las Instituciones de Educación Superior
}

Sally Barón Mercado Fundación Universitaria Colombo Internacional

Colombia

Ana Cantillo Orozco Universidad Tecnológica de Bolívar Colombia

Edgardo Martínez Tapia Fundación Universitaria Colombo Internacional Colombia 


\title{
Desafíos de la Responsabilidad Social Corporativa en las Instituciones de Educación Superior
}

\author{
Sally Barón Mercado ${ }^{(\mathbb{D})}$, Ana Cantillo Orozco ${ }^{2}$ (D) y Edgardo Martínez Tapia ${ }^{1(D)}$ \\ ${ }^{1}$ Fundación Universitaria Colombo Internacional, Colombia \\ ${ }^{2}$ Universidad Tecnológica de Bolívar, Colombia
}

acceso $\odot$ abierto

Para citaciones: Barón, S., Cantillo, A., y Martínez, T. (2020). Desafíos de la responsabilidad social corporativa en las instituciones de educación superior. Panorama Económico, 28(2), 116-128.

https://doi.org/10.32997/pe-2020-2693

Recibido: 04/11/2019

Aprobado: 02/03/2020

Autor de correspondencia:

Sally Barón Mercado

sbaron@unicolombo.edu.co

Editor: Andrés Escobar Espinoza

Universidad de Cartagena, Colombia

Tipología IBN Publindex:

Artículo de Reflexión

Copyright: (C) 2020. Barón, S., Cantillo, A., y Martínez, T. Este es un artículo de acceso abierto, distribuido bajo los términos de la licenciahttps://creativecommons.org/licenses/ by-nc-sa/4.0/ la cual permite el uso sin restricciones, distribución y reproducción en cualquier medio, siempre y cuando que el original, el autor y la fuente sean acreditados.

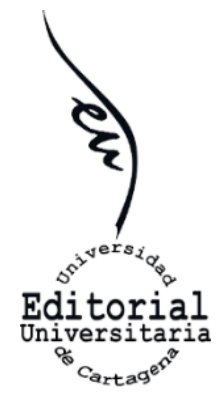

\section{RESUMEN}

El presente documento analiza las prácticas de Responsabilidad Social Corporativa en las Instituciones de Educación Superior (IES) y su relevancia en el actual periodo de acuerdos de paz entre el Estado y las Fuerzas Armadas Revolucionarias de Colombia (FARC). La Responsabilidad Social Corporativa puede promover la transformación social de las comunidades, mediante la cooperación y, la integración de las comunidades en los procesos de formación académica para fortalecer la dinámica económica regional. Finalmente, las estrategias de Responsabilidad Social Corporativa pueden fortalecer los procesos misionales de las Instituciones de Educación Superior, brindado el impulso necesario para promover tanto el liderazgo comunitario como las iniciativas de desarrollo social y económico en las comunidades vulnerables.

Palabras clave: Responsabilidad social corporativa; desarrollo local; Instituciones de Educación Superior.

JEL: L30, M10, M14.

\section{Corporate social responsability challenges for Higher Education Institutions}

\section{ABSTRACT}

This article analyzes the practices of Corporate Social Responsibility in Higher Education Institutions (HEIs) and their relevance in the current period of peace agreements between the State and the Revolutionary Armed Forces of Colombia (FARC). The Corporate Social Responsibility can promote the social transformation of the communities through cooperation and the integration of the communities in the academic training processes to strengthen the regional economic dynamics. Finally, Corporate Social Responsibility strategies can strengthen the missionary processes of Higher Education Institutions, providing the necessary impetus to promote both community leadership and social and economic development initiatives in vulnerable communities.

Key words: Corporate Social Responsibility; local development; Higher Education Institutions.

JEL: L30, M10, M14 


\section{INTRODUCCIÓN}

Los aportes de la educación superior en Colombia en el marco de los procesos que constituyen lo que se ha denominado como postconflicto, se han desarrollado de cara a dos ejes de desarrollo político; aquellos fenómenos derivados de los intereses institucionales y aquellos derivados de los intereses sociales. Esto en cuanto, las distintas reformas educativas han posibilitado en gran medida los procesos encaminados a la reconstrucción del tejido social por medio del desarrollo de nuevas perspectivas epistémicas de abordaje o reconocimiento de las comunidades vulnerables.

Es necesario entonces, la creación de espacios académicos sostenidos sobre la inclusión social, política y cultural, la construcción de amplios y flexibles modelos académicos que puedan ser capaces de brindar a través de un proceso educativo una verdadera re significación del sujeto, entendiéndose este como un sujeto activo de cambio social en el marco de un contexto educativo para la paz (Pachón, 2008).

Esta transformación de los procesos académicos, debe tener en cuenta "la formación, sensibilización y concientización de toda la comunidad académica" (Pachón, 2018, p.300), permitiendo la generación de ambientes inclusivos, con conocimiento de los procesos de paz reparación y conciliación dentro del ámbito educativo.

\section{ANTECEDENTES}

La Responsabilidad Social Corporativa (RSC), nace a partir de la realización del Foro Económico Mundial, que tuvo lugar en Davos (Suiza) en 1999, donde más de 250 líderes mundiales se reunieron para analizar las problemáticas de tendencia mundial que afectan el desarrollo de las naciones. Así mismo, las Instituciones de Educación Superior (IES) con la colaboración de las Naciones Unidas pueden aportar de forma significativa alternativas para enfrentar los desafíos de la sociedad actual. La Organización de las Naciones Unidas (ONU, 2012) explica cómo el denominado Pacto Mundial buscaba en su momento aprovechar las acciones y la fuerza colectiva de la empresa, a fin de contribuir a la solución de los retos planteados por la globalización. En tal razón, sector privado y otras entidades pueden aportar al crecimiento de una economía sostenible e inclusiva. Este punto de vista, permite sentar las bases para que las IES sean socialmente responsables, teniendo en cuenta principios que rigen aspectos referentes a la defensa de los derechos humanos, la protección de los ecosistemas y las medidas necesarias para combatir la corrupción. La Comisión de las Comunidades Europeas plantea en 2001, los lineamientos sobre RSE y la RSE consiste, citado por Martínez (2014), en la "integración voluntaria, por parte de las empresas, de las preocupaciones sociales y medioambientales en sus operaciones comerciales y relaciones con sus interlocutores" (p. 7). Igualmente, establece las políticas que pueden seguir las organizaciones para su cumplimiento. Así mismo, la estrategia denominada Europa 2020, promovida por los países que integran la Unión Europea, incluye el papel de la educación en la integración de la RSE, tal como se observa en las conclusiones de la Comisión de las Comunidades Europeas (2011):

El desarrollo de la RSE exige nuevas capacidades y cambios en los valores y los comportamientos. Los Estados miembros pueden desempeñar un importante papel animando a los centros educativos a integrar la responsabilidad social de las empresas, el desarrollo sostenible y la ciudadanía responsable en los programas 
educativos pertinentes, en particular en la educación secundaria y la enseñanza universitaria. (p. 22)

Lo anterior implica como, las IES aportan también a la adquisición de valores, conocimientos y competencias, mediante la participación de las personas en la generación de soluciones que impacten en lo político, lo social y lo económico, permitiendo así las transformaciones sociales que se esperan. Gaete (2011), refiriéndose a los alcances teórico-prácticos de la RSE, propone tres enfoques en su análisis: El primero, un enfoque gerencial o directivo que se orienta hacia el análisis del impacto de las acciones de las universidades en los stakeholders o partes interesadas. El segundo, un enfoque transformacional que se orienta hacia las contribuciones de las universidades al debate sobre la construcción de mejores sociedades. El tercero, un enfoque normativo que apunta a la relación que se configura entre el Estado y la sociedad, en cuanto a la aceptación de valores y principios universales.

Ospina (2003) propone cuatro aspectos principales asociados a la responsabilidad social. Primero, un aspecto laboral que expone el cumplimiento de normas laborales, desde los niveles jerárquicos hasta los trabajadores, mediante códigos de conducta basados en los principios propuestos por la Organización Internacional del Trabajo (OIT). Segundo, un aspecto económico, desde el cual se deben elaborar cuentas transparentes, abiertas al público, e inversiones basadas en responsabilidad social. El tercero, se refiere a lo ambiental que apunta a un respeto del medio ambiente, a través del uso de certificaciones de calidad ecológicas y sellos que permitan conocer por parte del consumidor el contenido y elaboración de los productos. Como último, está el aspecto social, el cual implica la inversión de una parte de las ganancias en proyectos que impacten en comunidades menos favorecidas, mejorando su calidad de vida.

En este sentido, Porter y Kramer (2006) plantean que estos aspectos hacen parte de la práctica de RSC, permitiendo la medición de indicadores de cobertura y de gestión, como es el caso de las rendiciones de cuentas de parte de las universidades públicas para su correcta integración. Cabe anotar que las organizaciones, desde la perspectiva de la norma internacional ISO 26000 (2010), deben rendir cuentas por los impactos producidos en cada uno de los aspectos de actuación: económico, social o ambiental, es decir estar atentos en su prevención o en su defecto evitar repetir impactos negativos en el sector externo. La necesidad de fortalecer dichos compromisos con la ciudadanía de forma activa, juega un papel fundamental en el análisis conceptual de la RSC. Para la promoción de estos en el ámbito local, con miras a lograr un desarrollo sostenible, tal como cita Gaete (2011), es importante contar con la participación voluntaria de distintos actores dentro del ámbito universitario (estudiantes, docentes, entre otros) desde una visión ética, propiciando la generación de un sentido de ciudadanía que proporcione servicios sociales a la comunidad.

Recale, Cantero y Jara (2012) sostienen que los espacios de educación superior y la estrategia corporativa están siendo trabajados para facilitar la inserción e integración de todos los procesos en la construcción de sociedades competitivas económicamente. La responsabilidad social corporativa incluye incorporar desde la perspectiva social las herramientas de dirección estratégica, gobierno corporativo, certificación, inversión social, TICS, marketing social y balance social adaptables a las exigencias del mercado 
actual. De esta forma, las universidades para responder a las necesidades del entorno socio-político, han venido transformando sus procesos en cuanto gestión, docencia, investigación y extensión para adaptarlas a las exigencias competitivas y la realidad social, que en el caso de Colombia tiende a ser relevante para generar una verdadera transformación social. La universidad, desde este punto de vista, debe orientarse hacia una dirección más activa en la difusión democrática, que se retroalimente permanentemente con el entorno, abriendo las puertas a todos los actores sociales y promoviendo el fortalecimiento de un pensamiento crítico en las personas. Esta visión muestra la importancia que tiene la RSC en el proceso de consolidación de la relación entre universidad y sociedad (Llopis, 2010).

Desde la década de los sesenta, a raíz del conflicto armado colombiano, las universidades del país se convirtieron en el escenario ideológico para generar desarrollo a partir de la generación del conocimiento; paralelo a esta realidad, se iniciaron acciones para promover escenarios de paz y sin lugar a dudas han tenido incidencia en las instituciones para seguir aunando esfuerzos con mayor inversión social, seguridad, así como una apuesta hacia la educación inclusiva como camino alterno para la construcción de paz, siendo posible desde el conocimiento y la comprensión integral de la población desde lo colectivo, para transformar los aspectos económicos de los que emerge la violencia en oportunidades de paz. Para el caso particular de Colombia, es imprescindible tener en cuenta la experiencia centroamericana en El Salvador y en Nicaragua, puesto que en estos países la superación de los conflictos se dio de manera escalonada y desescalada, con la participación de los grupos armados, los cuales posteriormente tomaron parte activa en las etapas de reconstrucción y de reconciliación (Delgado et al., 2008).

En Colombia, la Ley 30 de 1992 regula la Educación Superior (Congreso de Colombia, 1992), definida como un servicio público y establece que:

La Educación Superior es un proceso permanente que posibilita el desarrollo de las potencialidades del ser humano de una manera integral, se realiza con posterioridad a la educación media o secundaria y tiene por objeto el pleno desarrollo de los alumnos y su formación académica o profesional. (p. 1)

Por lo anterior, las IES de Colombia tienen incidencia histórica, como ejes fundamentales, en los procesos de reincorporación para los excombatientes con la potencialización de iniciativas de paz y nuevos ejercicios de ciudadanía. En especial, el Observatorio de Procesos de Desarme, Desmovilización y Reintegración considera que el empeño de los excombatientes permite consolidar las iniciativas ciudadanas, encontrando, como una vía, la formación profesional universitaria (ODDR, 2013). En este sentido, Pachón, Gómez y Méndez (2015) consideran que las instituciones de educación superior pueden ser alternativa de transformación social y de inclusión para la formación de personas, bajo "lineamientos de pluralidad democrática, paz sostenible y bienestar general de las comunidades" (p.83). En concordancia con lo planteado por el MEN, estos autores agregan al respecto:

Estos lineamientos han servido de base axiológica para estructurar el Plan Decenal de Educación (2006 - 2016), desarrollado desde el Ministerio de Educación Nacional (MEN). En materia de educación superior, el plan establece en el Título II, la "Educación en y para la paz, la convivencia y la Ciudadanía", proponiéndose 
adelantar acciones que le permitan al Estado involucrarse de manera más profunda en los procesos de desmovilización, reintegración y reconciliación a través de la educación (Pachón, Gómez y Méndez, 2018, p. 83).

Asimismo, la OCDE (2012) y el Banco Mundial, acerca de la Educación Superior en Colombia, se sostiene lo siguiente:

El sistema actual de educación superior (estructurado en torno a la Ley 30 de 1992, aunque no totalmente), enfrenta enormes retos de calidad, necesita un modelo de financiación más equitativo y universal y un diseño de sistema menos confuso para el país, requiere mayor fiabilidad y transparencia y mayor eficiencia en la gestión institucional de la educación superior, a fin de proporcionar una educación consistente con el contexto nacional y regional y en consonancia con el mundo. ( $p$. 35)

De lo anterior, se entiende la importancia de que las instituciones estrechen lazos cada vez más fuertes entre sí; esto debe incluir, por supuesto a las universidades y al sector empresarial, mediante procesos de inversión en áreas de investigación, la toma de decisiones importantes y la garantía sobre transparencia en los procesos, lo cual debe traducirse en la entrega de cuentas claras sobre la gestión emprendida y los recursos empleados, así como en las acciones adelantadas dentro de los procesos de internacionalización (OCDE \& Banco Mundial, 2013). Lo anterior, según se establece en el Índice de inclusión social (Ministerio de Educación Nacional, 2017) es de vital importancia, puesto que:

Busca potenciar y valorar la diversidad (entendiéndola y protegiéndola, lo cual implica garantizar, según el contexto colombiano, las identidades y particularidades de los estudiantes), promover el respeto a ser diferente y facilitar la participación de la comunidad dentro de una estructura intercultural, favoreciendo así la cohesión social, que es una de las finalidades de la educación. (p.18)

De allí que el proceso de transformación a nivel de educación superior, se estructura en cuatro dimensiones fundamentales: en primer lugar, la pertinencia de lo académico, respondiendo a las necesidades del contexto social; en segundo lugar, la necesidad de transformar una comunidad, entendiendo la importancia del conocimiento, pero traducido éste en desarrollo, expansión, riqueza e inclusión social; en tercer lugar, la prevalencia y defensa de los derechos humanos; finalmente, desde la órbita constitucional, la educación cumple una función de tipo social, pues a través de esta se busca acceder al conocimiento, a la ciencia, a la técnica, y a los demás bienes y valores de la cultura. Esto da como resultado la base de una educación para todos. Por lo que las Instituciones de Educación Superior (IES) deben crear espacios y programas en los que se tenga en cuenta esta población desmovilizada, para garantizarle el acceso a la educación (ODDR, 2010).

\section{Responsabilidad Social y su rol en la Instituciones de Educación Superior}

De este compromiso social ha sido partícipe la Fundación Universitaria Colombo Internacional, como institución de educación superior desde el año 2004, con la puesta en marcha de programas profesionales bilingües, como lo son: Derecho, Contaduría Pública, Licenciatura en Educación, Administración de Empresas, Ingeniería de Sistemas, 
Ingeniería de la Calidad y Administración de Empresas Turísticas. Esto ha sido una apuesta para que la comunidad del Sector Rojo participe en el proceso de Educación Bilingüe. UNICOLOMBO, como institución de formación académica de carácter superior y de gran impacto en la comunidad, dirige en un esfuerzo coordinado desde todos los ámbitos de gestión administrativa las cuales la constituyen la actividad docente, investigativa y de extensión a lograr una vinculación positiva y de impacto con el sector Foco Rojo en entendido que este constituye el marco geográfico de sus actividades de formación académica.

El propósito de Unicolombo, es consolidarse en el año 2030, como una de las más importantes instituciones de educación superior a nivel nacional de acuerdo al grupo poblacional en el cual se proyecta y el contexto internacional. En tal medida si misión evidencia el nivel de las expectativas sociales, las prioridades internas y las políticas gubernamentales con oferta de becas de bicentenario y programas Ceres, que tienen como objetivo disminuir las brechas de la desigualdad económica y social en Cartagena. Como lo señala Domínguez (2009), las universidades deben incorporar de manera necesaria dentro de sus estrategias las preocupaciones sobre lo social, el medio ambiente y los derechos humanos. De allí que el modelo de RSC en las universidades se pone en práctica por medio de una serie de principios y valores que integran cuatro procesos claves: Gestión, Docencia, Investigación y Extensión.

Figura 1. Modelo de RSC, Fundación Universitaria Colombo Internacional

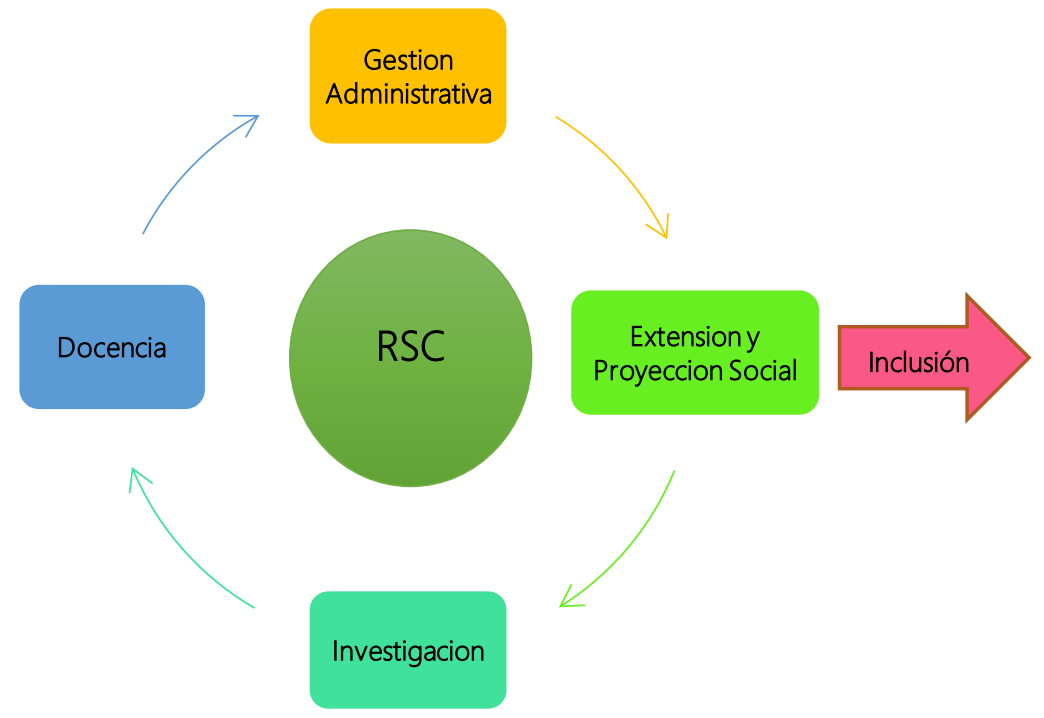

Fuente: Domínguez (2009)

Cabe anotar que, en los contextos del posconflicto, se ha generado la necesidad de incorporar política basadas en RSE, de forma estratégica para luchar contra fenómenos históricos como la pobreza, permitiendo la participación de personas que le apuestan a factores como la educación, la reconciliación y la inclusión de grupos minoritarios que buscan recuperar la dignidad permitiendo que víctimas o excombatientes jueguen un papel protagónico. La Fundación Universitaria Colombo Internacional se orienta hacia el bienestar social de las comunidades 1, 2 y 3 de Cartagena, promoviendo mejoras en la calidad de vida de los jóvenes en condiciones de vulnerabilidad; este propósito busca 
estimular la participación activa de la sociedad civil de la zona Foco Rojo, así como de establecer políticas de mejoramiento en materia de educación, inclusión, cultura, inversión social y física. Así los jóvenes se convierten en participes de proyectos sociales de gran impacto para la ciudad de Cartagena, los cuales buscan generar un viraje paradigmático que posibilite un cambio en el marco normativo del sistema educativo y derive con esto; en una educación superior de naturaleza inclusiva y democrática.

Desde esta perspectiva de inclusión, la estrategia apunta hacia la lucha por la exclusión social que afronta los aspectos diferenciales causados por factores materiales, económico, políticos y culturales, por ejemplo, el mercado laboral y la permanencia de derechos fundamentales y elementos simbólicos y subjetivos, como el rechazo, la indiferencia y la invisibilidad, como factores que atentan contra la identidad de las personas (MEN, 2014). Sobre esto, el Estado colombiano, en cabeza del MEN, define la educación inclusiva como un mecanismo para combatir toda forma de exclusión social. En este sentido el MEN, citado por Universidad Pontificia Javeriana (2016), aclara:

Es decir, como una estrategia parta afrontar ese proceso multidimensional caracterizado por una serie de factores materiales y objetivos, relacionados con aspectos económicos, culturales y políticos-jurídicos (ingresos, acceso al mercado de trabajo y a activos, derechos fundamentales), y factores simbólicos y subjetivos asociados a acciones determinadas que atentan la identidad de la persona (rechazo, indiferencia, invisibilidad). (p. 7)

De allí que dentro de los desafíos que deben asumir las IES consiste en actuar como agentes de paz para el proceso de transformación social que requiere el país, como proceso posterior al pos conflicto que sin lugar a dudas permite promover la cultura de la paz y aproximarnos a esa política inclusiva que señala el MEN. En este sentido la paz, se convierte en el referente de un orden social deseado en donde todos los actores en conflicto pueden participar desde prácticas legítimas y democráticas. En este sentido, la Universidad se constituye como un agente de cambio y transformación, en tanto institución de impacto social, no solo en pro de un desarrollo académico sino de formación del individuo integral como sujeto activo dentro del ámbito socio-político, es entonces, que la universidad debe por esencia propia constituirse en un en una de las bases necesarias para la superación del conflicto (Delgado et al., 2008).

A partir de lo anterior se presentan las estrategias de acción por la Fundación Universitaria Colombo Internacional y por la Universidad de Cartagena, ésta última como institución líder en la Costa Caribe, acreditada en alta calidad para promover la transformación social y la construcción de paz. 
Tabla 1. Estrategias de las IES para la Transformación Social y construcción de la Paz

\begin{tabular}{|c|c|c|}
\hline $\begin{array}{c}\text { Plan Nacional de } \\
\text { Desarrollo (2016-2019) }\end{array}$ & $\begin{array}{c}\text { Fundación Universitaria Colombo } \\
\text { Internacional }\end{array}$ & Universidad de Cartagena \\
\hline $\begin{array}{l}\text { "Cerrar las brechas en } \\
\text { acceso y calidad al sistema } \\
\text { educativo, entre individuos, } \\
\text { grupos poblacionales y } \\
\text { entre regiones, acercando } \\
\text { al país a altos estándares } \\
\text { internacionales y logrando } \\
\text { la igualdad de } \\
\text { oportunidades para todos } \\
\text { los ciudadanos" (Ministerio } \\
\text { de Educación Nacional, } \\
\text { 2015). }\end{array}$ & $\begin{array}{l}\text { La Educación Continua } \\
\text { está definida por los estudios } \\
\text { complementarios para la } \\
\text { construcción de conocimiento de } \\
\text { estudiantes, egresados, el sector } \\
\text { empresarial y la comunidad en } \\
\text { general a través de la diversificación } \\
\text { de Programas de Posgrado. } \\
\checkmark \quad \text { Implementación de } \\
\text { programas que realizan seguimiento } \\
\text { a los niveles de inserción estudiantil. } \\
\checkmark \quad \text { Los convenios de apoyo } \\
\text { financiero a la comunidad a través del } \\
\text { ICETEX, de Fundaciones y otros, para } \\
\text { el financiamiento de la educación } \\
\text { superior. Estas alianzas permiten que } \\
\text { se beneficie a poblaciones } \\
\text { vulnerables y de esta manera tengan } \\
\text { la posibilidad de acceder a la } \\
\text { educación superior Implementación } \\
\text { de Becas para personas de estratos } \\
\text { socioeconómicos } 1 \text { y } 2 \text { de la localidad. } \\
\checkmark \quad \text { Oferta de programas } \\
\text { tecnológicos e Interculturalidad a } \\
\text { través del fomento a un } \\
\text { reconocimiento del otro y de sí } \\
\text { mismo, en el marco del respeto, la } \\
\text { tolerancia y la interacción positiva con } \\
\text { otras realidades presentes } \\
\checkmark \quad \text { La looperación } \\
\text { Internacional permite la colaboración } \\
\text { con diversos actores de la sociedad: } \\
\text { Estado, Empresa Privada y sectores } \\
\text { sociales como ONGs y diversas } \\
\text { asociaciones que contribuyen a la } \\
\text { transferencia de recursos } \\
\text { económicos, físicos, tecnológicos, } \\
\text { académicos o humanos para el logro } \\
\text { de proyectos de desarrollo social en } \\
\text { beneficio de la sociedad y de la } \\
\text { institución. }\end{array}$ & 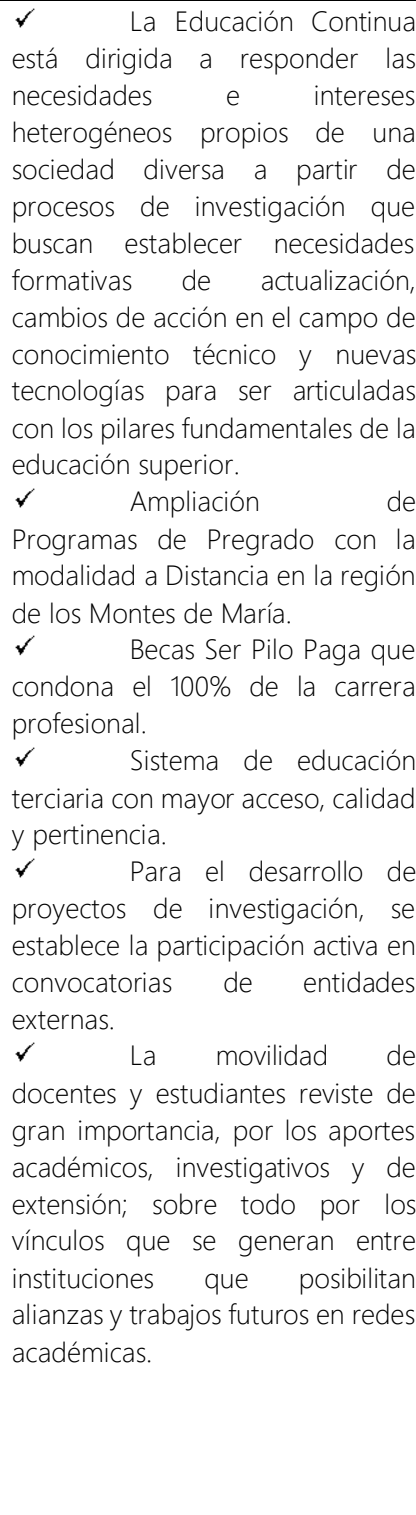 \\
\hline
\end{tabular}




\section{Continuación: Tabla 2}

\begin{tabular}{|c|c|c|}
\hline $\begin{array}{c}\text { Plan Nacional de } \\
\text { Desarrollo (2016-2019) }\end{array}$ & $\begin{array}{l}\text { Fundación Universitaria } \\
\text { Colombo Internacional }\end{array}$ & Universidad de Cartagena \\
\hline $\begin{array}{l}\text { "Formar una sociedad } \\
\text { integral moderna e } \\
\text { incluyente que respeta a sus } \\
\text { individuos, que genera un } \\
\text { crecimiento sostenible y un } \\
\text { estado que hace efectivo el } \\
\text { goce de sus derechos" } \\
\text { (Cañas, 2016). }\end{array}$ & $\begin{array}{l}\text { La Proyección Social } \\
\text { tiene como líneas de acción: } \\
\text { educación continua, convenios de } \\
\text { apoyo financiero para la } \\
\text { comunidad, asesorías, consultorías } \\
\text { y proyectos de impacto social. } \\
\checkmark \quad \text { El Centro de Desarrollo } \\
\text { Empresarial permite la interacción } \\
\text { entre el sector empresarial y la } \\
\text { comunidad en general, } \\
\text { atendiendo las necesidades del } \\
\text { tejido empresarial para el } \\
\text { fortalecimiento organizacional. } \\
\checkmark \quad \text { Las consultorías tienen } \\
\text { el propósito de influir en el } \\
\text { desarrollo socioeconómico de la } \\
\text { ciudad y la región. } \\
\checkmark \quad \text { La Investigación es } \\
\text { concebida como un conjunto de } \\
\text { procesos dirigidos a la } \\
\text { comprensión, interpretación y } \\
\text { explicación de la realidad cuyo } \\
\text { resultado es la producción, } \\
\text { aplicación, transformación y } \\
\text { avance de conocimiento científico. } \\
\checkmark \quad \text { La capacitación a } \\
\text { líderes comunitarios permite la } \\
\text { sensibilización de problemas y } \\
\text { alternativas de transformación } \\
\text { social para los jóvenes. } \\
\checkmark \quad \text { La enseñanza de } \\
\text { Idiomas Extranjeros permite el } \\
\text { desarrollo de competencias del } \\
\text { saber a }\end{array}$ & 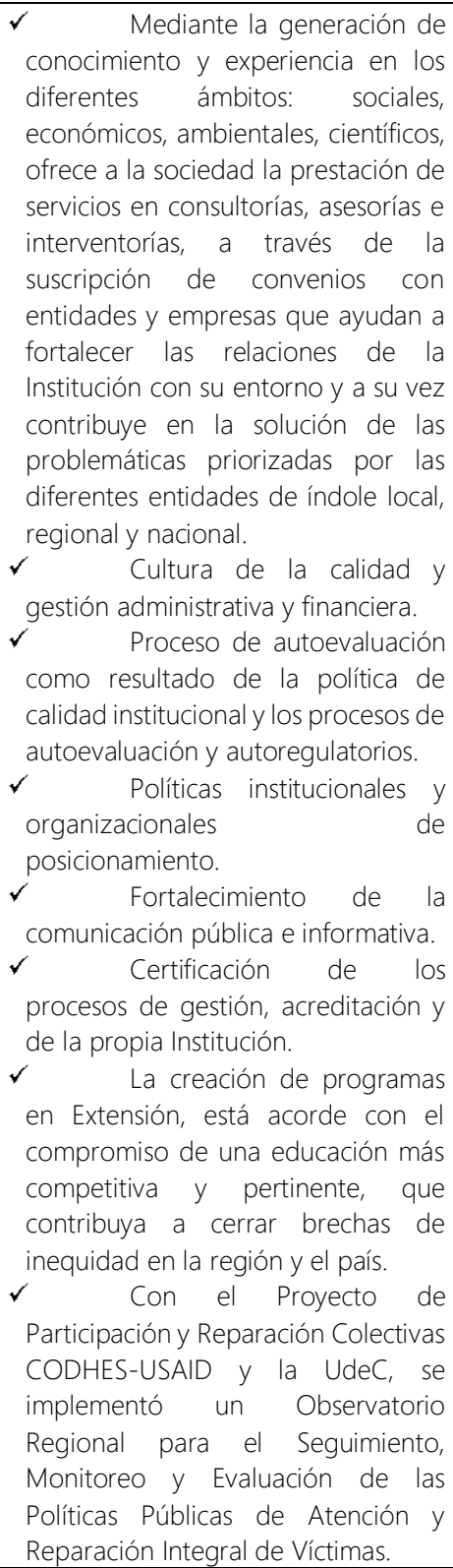 \\
\hline
\end{tabular}

Fuente: Elaboración propia con base en Unicolombo (2017) y Universidad de Cartagena (2017).

En este sentido, las iniciativas emprendidas por las IES generan un impacto positivo desde los principios organizacionales que les otorgan participación activa en la construcción de una educación inclusiva, competitiva y pertinente que les otorga mayores prerrogativas a los ciudadanos para disminuir las brechas de la inequidad en la región. El informe de rendición de cuentas (Universidad de Cartagena, 2016), señala que:

Es precisamente por medio de la extensión y proyección social, que la Universidad de Cartagena amplía su rango de acciones hacia la sociedad mediante determinados procesos, tales como la investigación, relaciones con los egresados, 
portafolios de servicios y demás herramientas que impacten de manera positiva el desarrollo económico y social de la comunidad en general. Así las cosas, desde la proyección social la Universidad puede y debe buscar un acercamiento en procura de un impacto social sostenible a través de proyectos en Convenio con entidades de orden local, departamental, regional y nacional para el cumplimiento de los fines del Estado. (p. 100)

En materia de integración, la Universidad de Cartagena adelanta proyectos como el Observatorio de organizaciones comunales y sociales para el desarrollo local (Universidad de Cartagena, 2016), a partir de capacitaciones a los líderes de distintas comunidades. Con la reciente creación de su Centro Tutorial en el municipio de San Juan Nepomuceno, denominado Universidad de la Paz, la institución apuesta a los procesos de inclusión para la población víctima del conflicto armado de la zona de los Montes de María, acogiéndose a las estrategias educativas lideradas desde el gobierno nacional. Entre las acciones realizadas, se encuentran investigaciones sobre conflicto y desplazamiento, procesos pedagógicos y formativos basados en los acuerdos de paz, conversatorios en distintas ciudades de la región, como: Cartagena, Magangué, San Juan Nepomuceno, el Carmen de Bolívar y Sincelejo (Sucre). Lo anterior con la participación de la población, Entidades Sin Ánimo de Lucro, Fundaciones, Armada Nacional y la comunidad académica. A partir de distintos convenios, se realizaron brigadas a víctimas en la zona de los Montes de María, Sincelejo y Cartagena, destacándose un total de 305 personas asesoradas. El propósito está enmarcado dentro de las acciones que persigue la construcción de la paz y constituyen expresiones de población objetivo, y alcance dentro de la RSU, entre otras variables.

Por otro lado, la Fundación Universitaria Colombo Internacional se destacan iniciativas planteadas dentro del marco de la RSC. Se destacan las aproximaciones para la implementación de un modelo que vincule los 4 ámbitos: Gestión, Docencia, Investigación, Extensión y Proyección Social, como parte del proceso de planeación estratégica contando con la disposición del recurso humano para crear compromiso, como paso necesario para implementar el proceso de RSU (Vallaeys, De la Cruz, \& Sasia, 2009). Entre los elementos incorporados por la institución para promover el modelo de Responsabilidad Social, se encuentran los principios filosóficos que determinan el propósito de orientación en la formación de profesionales abiertos al conocimiento universal, desarrollo científico, demandas cambiantes de la sociedad, proyectos que apunten a satisfacer necesidades de la comunidad.

Por otra parte, con relación a las matrículas académicas las personas que acceden por Plan Becario presentaron una variación para el año 2016 con el 23\% para beneficiarios del Programa Ceres y el 12\% para Becarios de Bicentenario. Para el año 2017, descendieron al 15\% para cada caso (Ceres y Bicentenario). Lo anterior, debido a los costos académicos, recursos académicos, infraestructura y naturaleza jurídica de la entidad. En la Universidad de Cartagena se cuenta con 181 cupos especiales reservados para quienes acceden por el sistema especial, y para el año 2016 un total de 4.656 estudiantes matriculados por sistema regular mientras que para el sistema especial con un total de 181 personas: 60 cupos para personas del Sur Bolívar, 22 cupos para las comunidades negras, 22 cupos para la representación de comunidades indígenas, 22 cupos para residentes en San Andrés y Providencia, 22 cupos zonas apartadas, 22 cupos 
zonas de difícil acceso o problemas de orden público y 11 cupos para personas reinsertadas

Tabla 2. Aspectos para potenciar la Responsabilidad Social Corporativa en la IES (Análisis DOFA)

\begin{tabular}{|c|c|}
\hline $\begin{array}{l}\text { Aspectos Positivos (que } \\
\text { aportan a la RSC en las } \\
\text { Instituciones de Educación } \\
\text { Superior) }\end{array}$ & $\begin{array}{l}\text { 1. Formación de profesionales bilingües } \\
\text { 2. Convenios y alianzas internacionales para movilidad académica } \\
\text { 3. Responsabilidad Proyección social } \\
\text { 4. Direccionamiento Estratégico } \\
\text { 5. Vinculación a redes académicas } \\
\text { 6. Recursos en TICS } \\
\text { 7. Marketing responsable } \\
\text { 8.Política de bienestar universitario } \\
\text { 9. Baja deserción de estudiantes. } \\
\text { 10. Proceso de autoevaluación permanente. }\end{array}$ \\
\hline $\begin{array}{l}\text { Aspectos para potenciar la } \\
\text { RS en la Institución) }\end{array}$ & $\begin{array}{l}\text { 1. Investigación } \\
\text { 2. Área encargada de hacer seguimiento a la RSC } \\
\text { 3. Sensibilización de la RS ante la comunidad académica } \\
\text { 4. Acreditación de los programas académicos } \\
\text { 5. Sensibilización de la educación inclusiva } \\
\text { 6. Asignaturas relacionadas con la RSC }\end{array}$ \\
\hline
\end{tabular}

Fuente: Elaboración Propia

Cabe anotar, que el problema de los bajos ingresos de gran parte de los estudiantes o sus familias impacta de forma directa en la posibilidad de asumir los costos financieros que se requieren para el ingreso a la educación superior. A pesar de que muchos logran acceder a los créditos que otorga el ICETEX, los recursos limitados impiden que el total, o la mayor parte de los estudiantes puedan acceder a ellos, evidenciando el papel de la responsabilidad social universitaria como generadora de espacios de vinculación.

Finalmente, aspectos como las políticas de movilidad que considera aquella entrante y saliente, tiene como propósito fortalecer la visibilidad de las instituciones, propiciar lazos de cooperación académica y redes de conocimiento nacionales e internacionales, además de facilitar los procesos de formación, investigación y asesoría a poblaciones vulnerables. Para resaltar la participación de las IES, se debe destacar su presencia en espacios de construcción de paz y demás aportes que permitan mitigar los efectos negativos ocasionados por la falta de acceso a educación. El reto más importante en el actual sistema de enseñanza superior colombiano, es la ampliación de un sistema educativo encaminado a la superación de problemas relacionados con las inequidades del acceso y la calidad. Desde esta perspectiva, se necesita la aplicación de un enfoque de naturaleza integral y multidimensional, para que sea posible garantizar la satisfacción de las necesidades básicas y contribuir efectivamente con el desarrollo económico tanto local como regional.

\section{CONCLUSIONES}

La Responsabilidad Social Corporativa (RSC) introduce el paradigma de la participación activa desde todos los ámbitos de funcionamiento de las IES en procesos dirigidos al mejoramiento social. Para esto, se articulan todos y cada uno de los procesos administrativos, de investigación, de actividad docente y de extensión para la generación de valor. Las políticas institucionales son el medio que permite la consecución de objetivos de la RSC a partir del fomento en valores, gestión del conocimiento, fortalecimiento del 
compromiso cívico y nuevos ambientes de aprendizaje bajo un enfoque ético que facilite la inserción e inclusión de una sociedad competitiva. Si lo que se busca, es responder de manera efectiva a las necesidades y problemas de tipo social, se deben iniciar sendos procesos de transformación, partiendo de la revisión de lineamientos institucionales en los que se vinculen elementos de inclusión educativa, con conocimiento de las diferencias en los contextos en los que impactan las universidades. Lo anterior permite que el quehacer universitario apunte hacia una proyección social basado en el eje de RSC.

En este sentido, la paz se convierte en el referente de un orden social deseado que involucra actores reinsertados, gobierno y sociedad civil. Es necesario que el Estado sea benefactor monetario de proyectos para la recuperación social y económica con mayor financiamiento en educación e inversión social. Lo anterior, permitirá un mayor progreso económico, reduciéndose la pobreza, tal como lo establecen los Objetivos de Desarrollo del Milenio. La implementación de prácticas de Responsabilidad Social Corporativa en las instituciones de educación superior, contribuye al proceso de transformación social: al responder a las necesidades del contexto social, al estimular la participación ciudadana, y fomentando la transformación del sistema educativo para atender la diversidad. Lo anterior traduce que las universidades asuman el liderazgo, desarrollo y sinergia frente a los escenarios de posconflicto en Colombia.

Las instituciones de educación superior pueden contribuir, desde su actividad de enseñanza, a la construcción de la paz, sobre todo si se comprende a la RSE como parte esencial de su misión y visión. Por tanto, se deben utilizar las herramientas adecuadas que garanticen el despliegue adecuado de las acciones y la sostenibilidad de las relaciones con las comunidades.

\section{REFERENCIAS}

Delgado, B. M., Vargas, P. J., Ramos, H. I. (2008). Los retos de la responsabilidad social universitaria: construyendo paz desde la universidad. Educación Superior y Sociedad, 13(2), 57-83.

Gaete, R. (2011). La responsabilidad social universitaria como desafío para la gestión estratégica de la Educación Superior: el caso de España. Revista de Educación, 355, 109-133. Recuperado de: http://www.revistaeducacion.educacion.es/re355 05.html

Llopis, E. (2010). Universidad y creación de valor para la sociedad. ¿Qué queremos de la universidad? (pp. 155-172). En M. De la Cuesta, C. De la Cruz, y J. Rodríguez (Coords.), Responsabilidad Social Universitaria. La Coruña: Netbiblo.

OCDE (2012). Reviews of National Policies for Education: Tertiary Education in Colombia. OECD and the International Bank for Reconstruction and Development/The World Bank. DOI http://dx.doi.org/10.1787/9789264180710-es

Pachón, W., Gómez, N., Méndez, A. (2015). Colombia: educación superior y post-conflicto. Revista Internacional de Aprendizaje en la Educación Superior, 2(2), 79-86. DOI: https://doi.org/10.37467/gka-revedusup.v2.1007

Porter, M., Kramer, M. (2006). Estrategia y Sociedad. Harvard Business Review America Latina, 84(12), 42-56

Recalde, H.A., Cantero, W., Jara, J. (2012). Importancia de la Responsabilidad Social Universitaria como desarrollo social de la ciudad de Coronel Oviedo. Revista Gestao Universitaria na America Latina, 5(4), 266-280. Recuperado de: https://www.redalyc.org/pdf/3193/319327517016.pdf

Restrepo, R. (2009). Responsabilidad Social Nuevas Teoría: Nuevas Prácticas. Medellín: Universidad de San Buenaventura. 
Unicolombo (2013). Plan de Desarrollo: Creciendo con calidad (2013-2018)

Universidad de Cartagena (2016). Rendición de Cuentas

Universidad de Cartagena (2017). Rendición de Cuentas

Vallaeys, F., De la Cruz, C., y Sasia, P. (2009). Responsabilidad Social Universitaria. Manual de primeros pasos. México: McGraw Hill. 